\title{
PROYECCIÓN DE TRABAJO EN EL INTERIOR DEL PAÍS Y FACTORES ASOCIADOS EN MÉDICOS RECIÉN COLEGIADOS DE LIMA, PERÚ 2010
}

\author{
Percy Mayta-Tristán 1,2,a, Christian R. Mejia3,4,b, Pedro Riega-Lopez ${ }^{2, c}$, \\ Leonardo Rojas-Mezarina ${ }^{4, d}$, Margarita Posso ${ }^{5, a}$, Edward Mezones-Holguín ${ }^{1,2, b}$
}

\begin{abstract}
RESUMEN
Objetivos. Estimar la prevalencia y factores asociados con la proyección de trabajo en el interior del país de médicos de Lima. Materiales y métodos. Se realizó un estudio de corte transversal en 782 médicos quienes fueron encuestados durante su proceso de colegiatura entre los meses de marzo y abril del 2010 . Se definió como proyección de trabajo en el interior del país si respondieron "interior del país" a la pregunta ¿dónde planea estar laborando en los próximos cinco años?. Se usó la regresión logística múltiple para encontrar los factores asociados. Resultados. La edad media de los participantes fue de 25 años, $54 \%$ fueron mujeres y $73 \%$ son de universidades privadas. Sólo $7,0 \%$ refirió proyectarse trabajar en el interior del país y el $0,5 \%$ en zonas rurales. No se encontró asociación con el género, lugar de nacimiento, tener familiares médicos, tipo de universidad, dominio del inglés, haber residido por más de un año en provincias y el año de realización del internado. En el análisis multivariado se encontró asociación con haber realizado el internado en un hospital del interior del país (OR:3,1; IC95\%:1,5-6,3), comunicarse en quechua (OR: 2,9; IC:1,4-6,1), que ambos padres nacieran en el interior del país (OR:2,8; IC:1,4-5,6); mientras que la proyección de un sueldo mensual superior a 5000 nuevos soles (OR:0,4; IC:0,2-0,8) y vivir con su familia nuclear o pareja (OR:0,3; IC:0,1-0,5) se asociaron en forma negativa. Conclusiones. Es baja la proyección de trabajo en el interior del país en los médicos colegiados en Lima. Algunos de los factores encontrados pueden ayudar a diseñar estrategias para revertir esta situación.
\end{abstract}

Palabras clave: Médicos; Migración interna; Distribución de médicos; Zonas rurales; Perú (fuente DeCS BIREME).

\section{LABOR PROJECTION IN THE COUNTRYSIDE AND ASSOCIATED FACTORS IN NEWLY COLLEGIATED PHYSICIANS IN LIMA, PERU 2010}

\begin{abstract}
Objectives. To estimate the prevalence and associated factors with the labor porjection in the countryside of physicians from Lima. Materials and methods. We conducted a cross-sectional study of 782 physicians who were surveyed during their affiliation at Peruvian Medical College during March and April 2010. Labor projection in the countryside was defined such as if they responded "inside the country" to the question "Where do you plan to be working in the next five years?" We used a multiple logistic regression model to find the associated factors. Results. The average age of the participants was 25 years, $54 \%$ were female and $73 \%$ graduated in private universities. Only $7.0 \%$ reported plans to work in the countryside and $0,5 \%$ in rural areas. No association with gender, place of birth, having physicians in their family, university type, the english efficiency, having resided for more than a one year in the provinces and the year of completion of internship was found. In the multivariate analysis, we found association with having made the internship inside the country (OR: $3.1,95 \% \mathrm{Cl}: 1.5-6.3$ ), communicate in Quechua (OR: 2.9, $\mathrm{Cl} 1.4-6.1$ ), both parents born in the countryside (OR: $2.8, \mathrm{Cl}: 1.4-5.6)$, while a monthly salary projection of more than $\$ 1780$ dollars (OR: $0.4 \mathrm{Cl}: 0.2-0,8)$ and living with their nuclear family or partner (OR: $0.3, \mathrm{Cl}: 0.1-0.5$ ) were negatively associated. Conclusions. Labor projection of work in the countryside of recently collegiated physicians from Lima is low. Some of the factors found can help to design strategies to reverse this situation.
\end{abstract}

Key words: Physicians: Internal migration; Physicians no distribution; Rural areas; Peru (source: MeSH NLM).

\footnotetext{
Escuela de Medicina, Universidad Peruana de Ciencias Aplicadas. Lima, Perú.

Instituto Nacional de Salud. Lima, Perú.

Asociación Médica de Investigación y Servicios en Salud. Lima, Perú.

Colegio Médico del Perú. Lima, Perú.

Universidad Autónoma de Barcelona. Barcelona, España.

a Médico salubrista; ${ }^{\text {b }}$ Médico epidemiólogo; ${ }^{\text {c }}$ Médico especialista en gestión en salud; d Médico especialista en administración en salud.
}

Recibido: 27-04-11 Aprobado: 15-06-11 


\section{INTRODUCCIÓN}

Los recursos humanos son la base de los sistemas de salud y su presencia determina en gran medida el éxito de su funcionamiento. En el Perú existe un serio déficit tanto de disponibilidad de los recursos humanos en salud (RHUS) a pesar que ha habido un incremento gradual en los últimos años ${ }^{(1)}$. Para el año 2007 el Ministerio de Salud (MINSA) estimó que se requieren entre 8446 a 15363 médicos -según el estándar usado- para satisfacer la demanda nacional en los servicios de salud del MINSA ${ }^{(2)}$.

Además de un problema de disponibilidad de RHUS en el Perú, existe una distribución inequitativa del mismo, por ejemplo, Lima Metropolitana concentra la mitad $(53,2 \%)$ de los médicos del país, la tasa de médicos por 10000 habitantes es 5,4 veces mayor que la región más pobre del país: Huancavelica (17,7 frente a 3,3) y casi el doble del promedio nacional, que para el año 2007 estaba por debajo de las metas regionales de RHUS planteadas por la Organización Panamericana de la Salud ${ }^{(3)}$. La distribución de los RHUS en el Perú es inversamente proporcional a los niveles de pobreza, la tasa de distribución de los médicos es de 3,7 a 1 entre los estratos no pobres y los más pobres ${ }^{(4)}$.

Por otro lado, en Lima -capital del país- se forma más del $45 \%$ de médicos a nivel nacional ${ }^{(5)}$ y se colegian hasta el $60 \%$. Existen reportes previos que han explorado una elevada intención de emigración al extranjero tanto de estudiantes de medicina de Lima ${ }^{(6)}$ como del interior del país que se encuentran entre las más altas de Latinoamérica, evidenciándose un flujo de provincia a la capital, de la capital al extranjero e incluso de la provincia al extranjero ${ }^{(7)}$. Esta situación nos lleva a preguntarnos si existe el flujo inverso, es decir, de la capital a la provincia. Sin embargo, no hemos encontrado estudios previos en el Perú sobre la intención de trabajo el interior del país o zonas rurales; conocer esta información puede permitir redistribuir mejor al recurso humano que potencialmente se incorporará al mercado laboral.

La intención de trabajar en las zonas más alejadas de la ciudad o la capital es influenciada por múltiples factores ${ }^{(8-11)}$. El desconocimiento de las expectativas laborales y los intereses personales de los RHUS ha condicionado un abordaje incompleto en las políticas implementadas para mejorar la distribución de los RHUS lo que contribuyó al fracaso de estas ${ }^{(12)}$. Por otro lado, las experiencias exitosas han tenido en cuenta el contexto socioeconómico, político e institucional, así como del mercado del trabajo en salud ${ }^{(13)}$.

Dado que existe una necesidad de cubrir la demanda de médicos que se requieren en el interior del Perú y que en Lima hay una mayor concentración de médicos, se deben implementar políticas que permitan mejorar esta situación, pero se requiere información sobre los intereses de los RHUS, por ello, este estudio busca explorar la prevalencia y factores asociados con la proyección de trabajo en el interior del país de médicos que se colegian en Lima en el año 2010.

\section{MATERIALES Y MÉTODOS}

Se realizó un estudio de corte transversal durante los meses de marzo y abril de 2010, durante el proceso de colegiatura de médicos en Lima, previa autorización del Colegio Médico del Perú (CMP) y consentimiento verbal de los participantes.

Dado que en Perú, se requiere obtener la colegiatura previamente al ejercicio de la profesión médica, los galenos recién egresados obligatoriamente realizan este procedimiento en las sedes descentralizadas del CMP, que para el caso de Lima es el Consejo Regional III Lima.

En promedio, cada año se colegian 1800 nuevos médicos, de los cuales entre $60 \%$ a $70 \%$ lo hacen en Lima y más del $95 \%$ durante el periodo de marzo y abril de cada año, coincidente con la primera convocatoria del Servicio Rural Urbano Marginal en Salud (SERUMS). Durante el proceso de colegiatura realizan una serie de trámites y reciben charlas sobre ética médica.

Se incluyó a todos los médicos que se colegiaron en Lima entre los meses de marzo y abril del 2010 y que decidieron participar voluntariamente de la investigación. Aquellos que devolvieron las encuestas incompletas o no llenaron las variables principales de estudio (proyección de trabajo en el interior del país o las que estuvieron asociadas en el análisis multivariado) no fueron incluidos en el análisis final.

Se elaboró un cuestionario con 55 ítems basado en estudios previos ${ }^{(7,14)}$ dividido en tres secciones (características generales, expectativas profesionales y percepciones sobre el trabajo médico), se realizó un piloto con 30 médicos que se colegiaron a finales de febrero de 2010 para evaluar la comprensión de las preguntas así como el tiempo del llenado del cuestionario que fue de aproximadamente $10 \pm 5$ minutos, luego de ello se hicieron ajustes en algunas preguntas.

La primera sección incluía datos generales como edad, género, lugar de nacimiento del encuestado y de sus padres, hospital donde realizó su internado, universidad de procedencia, presencia de familiares (abuelos, pa- 
dres o hermanos) médicos, dominio de idiomas (inglés o quechua), si ha vivido más de un año en provincias, con quien vive, año en que realizó el internado, entre otras. La segunda sección incluyó las expectativas profesionales a cinco años que incluían el lugar donde trabajar, realización de especialidad, cuanto deseaban percibir de sueldo mensualmente, entre otras. La tercera sección abordó las percepciones sobre diferentes escenarios para trabajar en provincias, como las diferencias de sueldo que deberían percibir con respecto al trabajo en Lima, en cuanto debería ser la diferencia, si deberían existir días libres y cuántos debían ser, entre otros.

Los médicos fueron invitados a participar durante los cursos sobre ética y aspectos legales de la medicina organizados por el Colegio Médico del Perú que debe recibir cada médico antes de colegiarse. Antes del inicio del curso, se les explicó el objetivo del estudio, el uso de sus resultados y en caso estén de acuerdo se le proporcionó la encuesta, la cual fue llenada en forma privada, anónima y autoadministrada.

Se definió como intención de trabajo al interior del país a la respuesta "provincia" ante la pregunta: "Dentro de 5 años usted planea estar laborando en (ubicación geográfica)". Se consideró vivir con la familia nuclear si es que declaraba que vivía con sus padres, hermanos o pareja.

La digitación se realizó en Microsoft Excel ® por un personal contratado ad hoc. Dos investigadores participaron del control de calidad de la base de datos. No se incluyeron en el análisis final las observaciones que tenían falta de respuesta en la variable de interés o los principales factores asociados estudiados; se evaluó los datos de los sujetos excluidos del análisis con aquellos que sí fueron incluidos para evaluar si habían diferencias en la edad, género y universidad de procedencia.

El análisis se realizó usando el paquete estadístico STATA 9.0 (STATA Corp, TX, USA). Se evaluó la normalidad de las variables numéricas por medio de la prueba de Shapiro-Wilk, comprobándose la ausencia de esta. A nivel descriptivo se usó la mediana y rango intercuartílico y frecuencias absolutas y relativas, para el caso de variables numéricas y categóricas, respectivamente. En el análisis bivariado, para evaluar la asociación entre las variables categóricas y numéricas con la proyección de trabajo al interior del país a cinco años, se utilizaron las pruebas de chi-cuadrado y la suma de rangos de Wilcoxon; respectivamente. Se consideró un $p<0,05$ como significativo. Se calculó el OR con su respectivo intervalo de confianza al 95\%. Con aquellas variables en las que el intervalo de confianza del OR no incluía la unidad, se desarrolló un análisis multivariado por medio de un modelo de regresión logística binaria, cuya bondad de ajuste fue evaluada por medio de la prueba de Hosmer-Lemeshow.

\section{RESULTADOS}

De un universo de 1020 médicos que se colegiaron en Lima durante el 2010, 888 lo hicieron entre marzo y abril y participaron de los cursos de ética médica que dictó el CMP, de ellos 25 se negaron a participar del estudio y 81 fueron excluidos por no haber completado las variables de interés, quedando para el análisis 782 (Figura 1). No hubo diferencias de edad, género, universidad de procedencia entre las personas que fueron excluidas e incluidas en el análisis final $(p>0,05)$.

\section{CARACTERISSTICAS GENERALES}

De los 782 médicos que participaron del estudio; 424 (54\%) fueron de género femenino, la edad promedio fue 25 años (rango 20-45), asimismo, el 94\% eran solteros y el $92 \%$ no tenía hijos. Un poco más de siete de cada diez de ellos nació en Lima Metropolitana o el Callao; no obstante, el $94 \%$ egresó de alguna universidad de Lima. El internado médico fue realizado en el Ministerio de Salud por el $70 \%$ de los encuestados, $88 \%$ lo realizaron en Lima y el $96 \%$ en el año 2009. Las características socio educativas de la población se pueden observar en la Tabla 1.

\section{EXPECTATIVAS PROFESIONALES Y LABORALES}

El $11 \%$ de los encuestados manifestaron que después de cinco años de colegiarse planeaba laborar en un trabajo, el $69 \%$ en dos trabajos, el $16 \%$ en tres trabajos y $4 \%$ en más de tres trabajos a la vez. El 96,7\% (668) piensan postular a la residencia médica, $341(51,0 \%)$ refieren la intención de hacerlo en el extranjero, 75 de ellos refieren también poder hacerlo en el Perú, 437 (65,4\%) manifestaron hacer su residencia en el Perú, de ellos sólo $4(1,1 \%)$ manifestaron hacerlo en una provincia fuera de Lima. Así mismo, planean tener un sueldo mínimo mensual promedio de $3762 \pm 237$ nuevos soles.

\section{TRABAJO EN EL INTERIOR DEL PAÍS}

Solo 55 de los 782 médicos evaluados, se habían proyectado trabajar en el interior del país después de cinco años y de ellos sólo cuatro $(0,5 \%)$ planean hacerlo en zonas rurales. Cuándo se interrogó sobre algunas condiciones que deberían de cumplirse para realizar actividades en el interior del país; el $94 \%$ manifestaron que su sueldo debería ser más alto del que podría percibir en Lima, en ese sentido el sueldo promedio requerido sería 


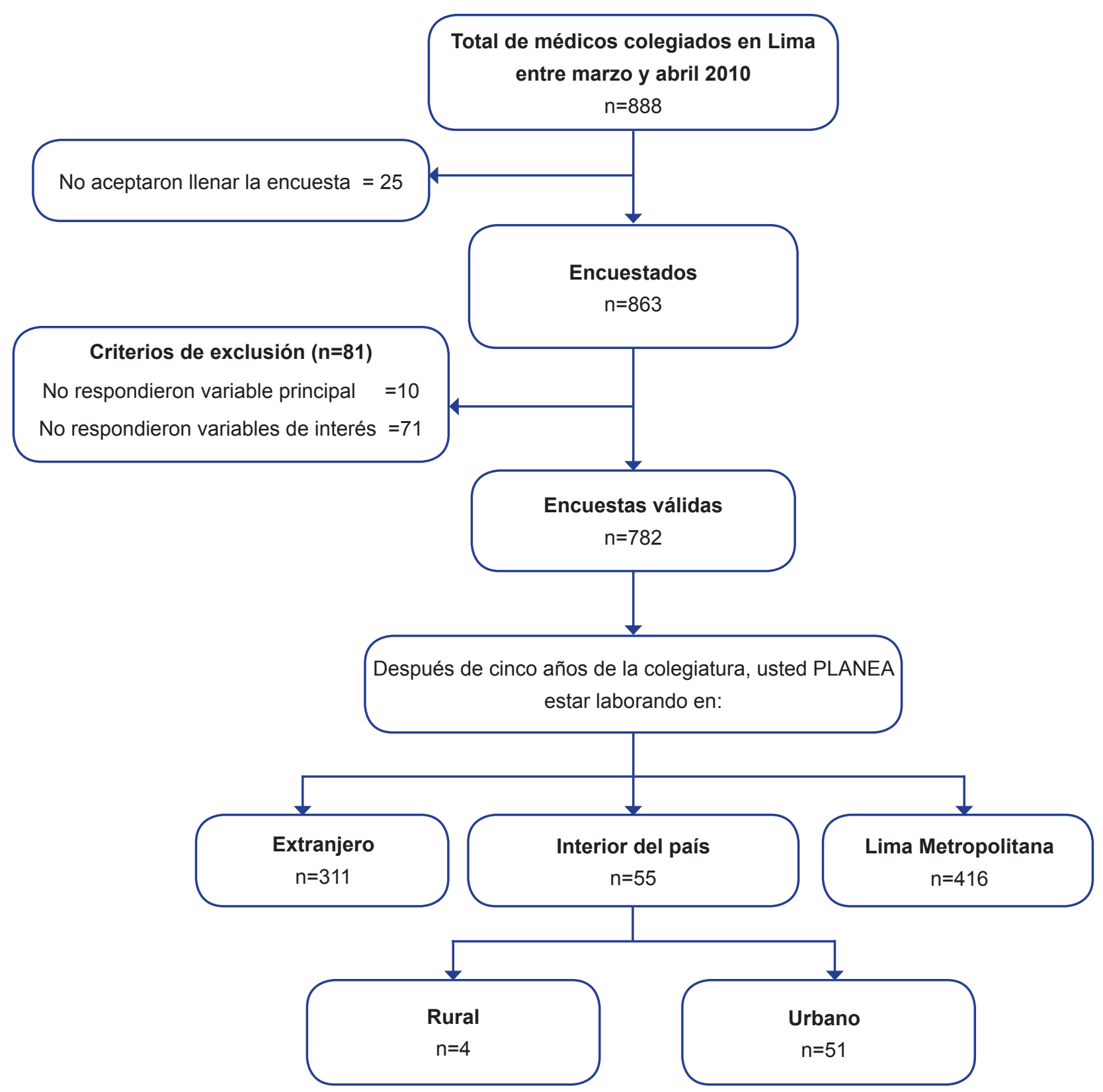

Figura 1. Flujograma de participación en el estudio.

de 7961,8 \pm 350,0 nuevos soles. Asimismo, en el caso de laborar en el interior del país, la media de días libres que desearían tener sería de 7,9 $\pm 0,3$ días por mes.

\section{ANÁLISIS BIVARIADO}

Al tomar la intención de laborar en el interior del país, se encontró que esta se asociaba con el conocimiento de idioma quechua $(p<0,001)$, el hecho de que ambos padres hayan nacido en provincias $(p<0,001)$, el año cronológico de realización del internado $(p<0,001)$, el hecho de haber residido en el interior del país por más de un año $(p<0,001)$, la ubicación del hospital donde realizó su internado $(p=0,004)$ y el sueldo que desea percibir $(p=0,007)$. Otras variables exploradas no mostraron una asociación significativa (Tabla 2).

\section{ANÁLISIS MULTIVARIADO}

Con las variables que resultaron significativas en el análisis crudo, se desarrolló un modelo de regresión logística binaria considerando la intención de laborar en el interior del país como variable de respuesta. Se observó que el vivir con familiar nuclear o pareja (OR: 0,25, IC95\% 0,13-0,51; $p<0,001)$ y el deseo de percibir más de 5 mil nuevos soles en dos años se asociaron de manera 
Tabla 1. Características socio-educativas de los recién egresados de universidades de Lima, 2010.

\begin{tabular}{|c|c|c|}
\hline Características & $\mathbf{n}$ & $(\%)$ \\
\hline \multicolumn{3}{|l|}{ Género } \\
\hline Masculino & 356 & $(45,6)$ \\
\hline Femenino & 424 & $(54,4)$ \\
\hline Edad & \multicolumn{2}{|c|}{$25 \pm 12,25^{\star *}$} \\
\hline \multicolumn{3}{|l|}{ Estado civil } \\
\hline Soltero & 733 & $(94,1)$ \\
\hline Casado & 34 & $(4,4)$ \\
\hline Conviviente & 12 & $(1,5)$ \\
\hline \multicolumn{3}{|l|}{ Tiene hijos } \\
\hline Sí & 62 & $(8,0)$ \\
\hline No & 714 & $(92,0)$ \\
\hline \multicolumn{3}{|l|}{ Lugar nacimiento } \\
\hline Lima o Callao & 549 & $(74,1)$ \\
\hline Interior del país & 182 & $(24,6)$ \\
\hline Extranjero & 10 & $(1,3)$ \\
\hline \multicolumn{3}{|l|}{ Universidad (ubicación) } \\
\hline Lima & 727 & $(94,1)$ \\
\hline Provincias & 18 & $(2,3)$ \\
\hline Extranjero & 28 & $(3,6)$ \\
\hline \multicolumn{3}{|l|}{ Tipo de universidad* } \\
\hline Universidad nacional & 177 & $(22,9)$ \\
\hline Universidad particular & 568 & $(73,5)$ \\
\hline \multicolumn{3}{|l|}{ Institución donde realizó el internado† } \\
\hline MINSA & 547 & $(70,4)$ \\
\hline EsSalud & 92 & $(11,8)$ \\
\hline Sanidades & 114 & $(14,7)$ \\
\hline Otros & 24 & $(3,1)$ \\
\hline \multicolumn{3}{|l|}{ Lugar donde realizó el internado } \\
\hline Lima & 689 & $(88,1)$ \\
\hline Provincia & 93 & $(11,9)$ \\
\hline \multicolumn{3}{|l|}{ Año que culminó el internado } \\
\hline 2009 & 753 & $(96,3)$ \\
\hline 2008 & 19 & $(2,4)$ \\
\hline$<2008$ & 10 & $(1,3)$ \\
\hline \multicolumn{3}{|l|}{ Familiares médicos } \\
\hline No & 348 & $(44,9)$ \\
\hline Sí & 426 & $(55,1)$ \\
\hline Padres, abuelos, hermanos & 201 & $(47,5)$ \\
\hline Otros familiares & 222 & $(52,5)$ \\
\hline \multicolumn{3}{|l|}{ Lugar nacimiento de sus padres } \\
\hline Ambos en Lima & 184 & $(23,5)$ \\
\hline Ambos en el interior del país & 397 & $(50,8)$ \\
\hline Uno en Lima & 201 & $(25,7)$ \\
\hline \multicolumn{3}{|c|}{ Vivió por más de un año en el interior del país } \\
\hline Sí & 271 & $(34,7)$ \\
\hline No & 511 & $(65,3)$ \\
\hline \multicolumn{3}{|l|}{ Con quien vive } \\
\hline Solo & 52 & $(6,7)$ \\
\hline Pareja & 48 & $(6,1)$ \\
\hline Padres y/o hermanos & 651 & $(83,3)$ \\
\hline Otros & 31 & $(3,9)$ \\
\hline \multicolumn{3}{|l|}{ Idiomas } \\
\hline Quechua & 56 & $(10,5)$ \\
\hline Inglés & 760 & $(97,2)$ \\
\hline Otro idiomał & 195 & $(24,9)$ \\
\hline
\end{tabular}

* Solo se consideró a los que estudiaron medicina en el Perú.

** Mediana \pm desviación cuartílica.

† MINSA: Ministerio de Salud del Perú, EsSalud: Seguro social del Perú, Sanidades: incluye hospitales de las fuerzas armadas y policiales.

$\ddagger$ Incluyen portugués, francés, chino, entre otros. negativa. Por su parte la ubicación del hospital de realización del internado en provincias ( $p=0,002$, OR: 3,09, IC95\% 1,51-6,27), el tener ambos padres oriundos del interior del país ( $p=0,003$, OR: 2,84, IC95\% 1,43-5,62) y el tener conocimiento del idioma quechua $(p=0,005$, OR: 2,89, IC95\% 1,38-6,05) se asocian positivamente (Tabla 2).

\section{DISCUSIÓN}

Solo uno de cada catorce médicos colegiados en Lima planea laborar en el interior del país luego de un periodo de cinco años de haber egresado y solo uno de cada 200 piensa hacerlo en zonas rurales, con esta tendencia, podríamos afirmar que continuará la distribución inequitativa de médicos, siguiendo una mayor concentración de médicos en Lima ${ }^{(15)}$. Por ello, es necesario tomar en cuenta los factores asociados con una mayor intención de trabajo en el interior del país para el diseño de estrategias que permitan una mejor redistribución del recurso humano.

El haber realizado el internado en un hospital del interior del país fue el factor más fuertemente asociado en el análisis multivariado, lo cual puede deberse a lo positivo de esta experiencia y la identificación de oportunidades de desarrollo personal, profesional o económico. Este resultado es consistente con múltiples estudios que demuestra que la formación profesional en escenarios rurales incrementa la posibilidad de retorno de los profesionales a esas zonas para ejercer su profesión ${ }^{(16-22)}$.

Sin embargo, cabe señalar que se ha encontrado que la exposición durante el pregrado al primer nivel de atención en zonas rurales ha tenido el efecto inverso en escenarios donde las deficiencias y limitaciones encontradas llevaron una reducción de la intención de trabajo en zonas rurales (Mayta-Tristán, datos no publicados).

Otro factor evaluado sobre la exposición a la realidad del interior del país, fue el haber vivido en regiones durante más de un año, pero no se encontró asociación significativa en el análisis multivariado. Esto refuerza la idea que no sólo es necesario exponer a los estudiantes al entorno en que se le desea ubicar, sino que es de importancia capital el tomar los cuidados en la implementación y características de la exposición para procurar una experiencia positiva que tenga el efecto deseado sobre los futuros médicos ${ }^{(13,23,24)}$.

Hablar el idioma quechua, segundo idioma oficial del Perú, así como que ambos padres hayan nacido en el interior del país, influyen y favorecen la intención de trabajar en regiones en los médicos que empiezan a 
Tabla 2. Factores asociados con la proyección de trabajo en el interior del país en médicos colegiados en Lima, 2010.

\begin{tabular}{|c|c|c|c|c|c|c|c|c|c|c|}
\hline \multirow{3}{*}{ Características } & \multicolumn{4}{|c|}{$\begin{array}{c}\text { Se proyectó trabajar en el } \\
\text { interior del país }\end{array}$} & \multicolumn{3}{|c|}{ Análisis Bivariado } & \multicolumn{3}{|c|}{ Análisis Multivariado** } \\
\hline & \multicolumn{2}{|c|}{ Sí } & \multicolumn{2}{|c|}{ No } & \multirow{2}{*}{ OR } & \multirow{2}{*}{ (IC95\%) } & \multirow{2}{*}{$\mathbf{p}$} & \multirow{2}{*}{ - OR } & \multirow{2}{*}{ (IC95\%) } & \multirow{2}{*}{$\mathbf{p}$} \\
\hline & $\mathbf{n}$ & $(\%)$ & $\mathbf{n}$ & $(\%)$ & & & & & & \\
\hline \multicolumn{11}{|c|}{ Ubicación del hospital del internado } \\
\hline Provincia & 14 & $(15,1)$ & 79 & $(84,9)$ & 2,80 & $(1,35-5,52)$ & 0,004 & 3,09 & $(1,51-6,27)$ & 0,002 \\
\hline Lima & 41 & $(6,0)$ & 648 & $(94,0)$ & & & & & & \\
\hline \multicolumn{11}{|l|}{ Se comunica en Quechua } \\
\hline Sí & 14 & $(25,0)$ & 42 & $(75,0)$ & 5,57 & $(2,59-11,41)$ & $<0,001$ & 2,89 & $(1,38-6,05)$ & 0,005 \\
\hline No & 41 & $(5,6)$ & 685 & $(94,4)$ & & & & & & \\
\hline \multicolumn{11}{|c|}{ Ambos padres nacieron en provincia } \\
\hline Sí & 43 & $(10,8)$ & 354 & $(89,2)$ & 3,78 & $(1,91-7,99)$ & $<0,001$ & 2,84 & $(1,43-5,62)$ & 0,003 \\
\hline No & 12 & $(3,1)$ & 373 & $(96,8)$ & & & & & & \\
\hline \multicolumn{11}{|l|}{ Sueldo que desea percibir * } \\
\hline S/.5000 ó más & 23 & $(4,9)$ & 442 & $(95,1)$ & 0,46 & $(0,25-0,84)$ & 0,007 & 0,43 & $(0,24-0,78)$ & 0,006 \\
\hline Menos de S/.5000 & 32 & $(10,1)$ & 285 & $(89,9)$ & & & & & & \\
\hline \multicolumn{11}{|c|}{ Vive con familia nuclear o pareja } \\
\hline Sí & 39 & $(5,6)$ & 660 & $(94,4)$ & 0,25 & $(0,13-0,50)$ & $<0,001$ & 0,25 & $(0,13-0,51)$ & $<0,001$ \\
\hline No & 16 & $(19,3)$ & 67 & $(80,7)$ & & & & & & \\
\hline \multicolumn{11}{|c|}{ Ha vivido en provincia más de un año } \\
\hline Sí & 36 & $(13,3)$ & 235 & $(86,7)$ & 3,97 & $(2,16-7,47)$ & $<0,001$ & $\dagger$ & & \\
\hline No & 19 & $(3,7)$ & 492 & $(96,3)$ & & & & & & \\
\hline \multicolumn{11}{|l|}{ Año que realizó el internado } \\
\hline 2009 & 46 & $(6,1)$ & 706 & $(93,9)$ & 0,17 & $(0,07-0,48)$ & $<0,001$ & $\dagger$ & & \\
\hline Antes del 2009 & 8 & $(27,6)$ & 21 & $(72,4)$ & & & & & & \\
\hline \multicolumn{11}{|l|}{ Lugar de nacimiento } \\
\hline Urbana & 46 & $(6,8)$ & 632 & $(93,9)$ & 0,22 & $(0,06-0,97)$ & 0,023 & & & \\
\hline Rural & 4 & $(25,0)$ & 12 & $(72,4)$ & & & & & & \\
\hline \multicolumn{11}{|l|}{ Domina idioma extranjero } \\
\hline Sí & 51 & $(6,7)$ & 711 & $(93,3)$ & 0,29 & $(0,09-1,23)$ & 0,046 & & & \\
\hline No & 4 & $(20,0)$ & 16 & $(80,0)$ & & & & & & \\
\hline \multicolumn{11}{|l|}{ Universidad de procedencia } \\
\hline Fuera de Lima & 48 & $(6,6)$ & 679 & $(93,4)$ & 0,60 & $(0,14-5,56)$ & 0,038 & & & \\
\hline Lima Metropolitana & 7 & $(15,2)$ & 39 & $(84,8)$ & & & & & & \\
\hline \multicolumn{11}{|l|}{ Género } \\
\hline Masculino & 26 & $(7,3)$ & 330 & $(92,7)$ & 1,07 & $(0,59-1,93)$ & 0,801 & & & \\
\hline Femenino & 29 & $(6,8)$ & 395 & $(93,2)$ & & & & & & \\
\hline \multicolumn{11}{|l|}{ Tiene familiares médicos } \\
\hline No & 25 & $(7,2)$ & 323 & $(92,8)$ & 1,02 & $(0,56-1,84)$ & 0,939 & & & \\
\hline Sí & 30 & $(7,0)$ & 396 & $(93,0)$ & & & & & & \\
\hline
\end{tabular}

ejercer. Ambas variables podrían representar una mayor identificación del médico con las necesidades del interior del país. Aproximaciones cualitativas sobre el trabajo en zonas rurales han mostrado evidencias de una mejor percepción de la atención por parte de los usuarios así como del personal de salud en aquellos que manejan el idioma quechua ${ }^{(25,26)}$. Una medida a explorar podría ser la enseñanza del quechua médico o una profundización de la salud intercultural como una medida para aproximar más a los estudiantes a las poblaciones más necesitadas del país.

Por otro lado, tenemos a los factores que estuvieron asociados a una menor intención de trabajo en el interior del país. Uno de ellos es el vivir con un familiar cercano o la pareja, que puede representar ya sea una carga emocional mayor, como también una mayor estabilidad a través del vínculo familiar ${ }^{27)}$. Por tanto, el no tener víncu- 
los familiares podría asociarse al desarrollo profesional fuera de lugar donde realizó sus estudios universitarios. En este aspecto, otros estudios han encontrado que los médicos solteros tienen más posibilidad de cambiar de trabajo y migrar que los casados ${ }^{(22)}$. En nuestro estudio no se encontró relación entre el género y la intención de trabajar al interior del país. Este resultado contrasta con otros en los que la migración obedece principalmente a factores distintos entre hombres y mujeres, siendo el aspecto económico más importante para el género masculino, y el aspecto familiar y el matrimonio para el género femenino ${ }^{(16)}$.

Finalmente, la asociación significativa entre un sueldo mayor a cinco mil nuevos soles y planear laborar en la capital, puede significar que los médicos esperan encontrar mayores oportunidades para obtener mejores ingresos económicos en la capital. Además de ello, los médicos reconocen que el trabajo en regiones amerita un mejor pago, y que de darse el caso de trabajar al interior del país, esperarían un sueldo correspondiente a 2,59 veces el sueldo promedio del médico en el país; sin embargo, la realidad suele ser distinta, donde los médicos que laboran en Lima en el MINSA ganan más que los que trabajan en provincias ${ }^{(28)}$. Este resultado es coherente con otros estudios en los que se evidencia que el incentivo económico es uno de los factores centrales para promover el trabajo de recursos humanos en zonas alejadas de las capitales, a pesar de esclarecer que como política única, es insuficiente ${ }^{(29)}$.

El estudio presenta limitaciones, en primer lugar no se encuestó a la totalidad de médicos colegiados en Lima y la encuesta se dio en un contexto previo a la exposición al Servicio Rural Urbano Marginal, experiencia en la que los factores del entorno pueden ejercer un papel de atracción o repulsión según lo positivo o negativo de la experiencia a vivir.

Si bien es cierto es importante conocer la predisposición de los médicos formados en Lima para trabajar en el interior del país, es necesario investigar este aspecto en los mismos médicos formados en las regiones, ya que de encontrarse una tendencia hacia movilizarse a las zonas más urbanas y comerciales, se evidenciará que el problema tiene aún mayor intensidad y con una tendencia opuesta a la esperable para lograr la reducción de la brecha entre la necesidad y disponibilidad de profesionales médicos en las regiones.

Esta información puede ser de utilidad para la implementación de políticas nacionales de salud que contribuyan a una adecuada distribución de profesionales de salud, y en particular de médicos, en todo el territorio nacional. Por ejemplo, el exponer durante el pregrado a los estudiantes formados en Lima a experiencias positivas en el interior del país. Existen algunas experiencias que se vienen dando en el país como los CUMIS ${ }^{(30)}$ o IRIS ${ }^{(31)}$ que son actividades de proyección social e investigación que realizan estudiantes de medicina en forma multidisciplinaria en áreas rurales, que permiten una exposición precoz y positiva a la realidad de la zonas rurales, el efecto que tienen sobre la intención de trabajo en el interior del país es un aspecto que debe ser evaluado y de ser efectivo, debe ser incentivado y desarrollado de una forma más estructural por las universidades.

En conclusión, la proyección a trabajar en el interior del país en un plazo medio de cinco años en médicos colegiados en Lima es muy baja, y los factores que la favorecen son el haber realizado el internado en un hospital de región, el dominar el idioma quechua y tener ambos padres nacidos en regiones; mientras que los factores que la reducen son una alta expectativa salarial y el vivir con la familia nuclear o la pareja.

\section{Contribuciones de Autoría}

PMT y PRL participaron en la concepción del estudio, MP, PMT y CRM diseñaron el estudio, CRM, PRL y LRM recolectaron los datos, PMT supervisó el avance del estudio, EMH y CRM realizaron el control de calidad de los datos y participaron del análisis de los datos junto a PMT, todos los autores interpretaron los resultados y participaron en la redacción y aportes críticos del artículo así como en la aprobación de la versión final a publicar.

\section{Fuente de Financiamiento}

Autofinanciado

\section{Conflictos de Interés}

Los autores declaran no tener conflictos de interés en la publicación de este artículo.

\section{REFERENCIAS BIBLIOGRÁFICAS}

1. Arroyo J. Los sistemas descentrados de recursos humanos en salud: el caso del Perú, 1990-2005. Cienc Saúde Coletiva. 2006;11(4):1063-72.

2. Perú, Ministerio de Salud. Estudio de la dotación de profesionales de la salud en los establecimientos del Ministerio de Salud. Lima: MINSA; 2007.

3. Organización Panamericana de la Salud. Metas regionales en materia de recursos humanos para la salud 2007-2015. Resolución Nro. CSP27/10. Washington DC: OPS; 2007.

4. Perú, Ministerio de Salud, Observatorio Nacional de Recursos Humanos en Salud. Situación y desafíos de los recursos humanos en salud. Gobernabilidad y desempeño con desarrollo humano. Lima: MINSA; 2005. 
5. Alva J, Verastegui G, Velasquez E, Pastor R, Moscoso B. Oferta y demanda de campos de práctica clínica para la formación de pregrado en estudiantes de ciencias de la salud en el Perú, 2005-2009. Rev Peru Med Exp Salud Publica. 2011;28(2):194-201.

6. Mayta-Tristán P, Dulanto-Pizzorni A, Miranda JJ. Low wages and brain drain: an alert from Peru. Lancet. 2008;371:1577

7. Mayta-Tristán P, Carbajal-Gonzalez D, Mezones-Holguín E, Mejia CR, Pereyra-Elías R, et al. Situación actual y perspectivas profesionales de los estudiantes de medicina de nueve países de Latinoamérica, 2008: estudio preliminar. CIMEL. 2010;15(1):3-8.

8. Rourke J. How can medical schools contribute to the education, recruitment and retention of rural physicians in their region? Bull World Health Organ. 2010;88(5):395-6.

9. Dieleman M, Harnmeijer J. Improving health worker performance: in search of promising practices. Geneva: World Health Organization; 2006

10. Serneels PM, Lindelow M, Montalvo JG, Barr A. For public service or money: understanding geographical imbalances in the health workforce. Health Policy Plan. 2007;22(3):128-38.

11. Peña S, Ramirez J, Becerra C, Carabantes J, Arteaga O. The Chilean Rural Practitioner Programme: a multidimensional strategy to attract and retain doctors in rural areas. Bull World Health Organ. 2010;88(5):371-8.

12. Lehmann U, Dieleman M; Martineau T. Staffing remote rural areas in middle- and low-income countries: A literature review of attraction and retention. BMC Health Serv Res. 2008;8:19.

13. Buchan J. What difference does ("good") HRM make? Hum Resour Health. 2004;2(1):e6.

14. Mayta-Tristán P, Dulanto-Pizzorni A. Prevalencia y factores asociados con la intención de emigración en internos de medicina de una universidad pública, Lima 2007. Rev Peru Med Exp Salud Publica. 2008;25(3):274-8.

15. Zevallos L, Pastor R, Moscoso B. Oferta y demanda de médicos especialistas en los establecimientos de salud del Ministerio de Salud: brechas a nivel nacional, por regiones y tipos de especialidad. Rev Peru Med Exp Salud Publica. 2011;28(2):177-85.

16. Smith JP, Thomas D. On the road: marriage and mobility in Malaysia. J Human Resour. 1998;33(4):805-32.

17. Rabinowitz HK, Diamond JJ, Markham FW, Paynter NP. Critical factors for designing programs to increase the supply and retention of rural primary care physicians. JAMA. 2001;286(9):1041-8.

18. Rabinowitz HK, Diamond JJ, Markham FW, Rabinowitz C. Longterm retention of graduates from a program to increase the supply of rural family physicians. Acad Med. 2005;80(8):728-32.

19. Brooks RG, Walsh M, Mardon RE, Lewis M, Clawson A The roles of nature and nurture in the recruitment and retention of primary care physicians in rural areas: a review of the literature. Acad Med. 2002;77(8):790-8.
20. de Vries E, Reid S. Do South African medical students of rural origin return to rural practice? S Afr Med J. 2003;93(10):789-93.

21. Dovlo D. Using mid-level cadres as substitutes for internationally mobile health professionals in Africa. A desk review. Hum Resour Health. 2004;2(1):7.

22. Rabinowitz HK, Diamond JJ, Markham FW, Hazelwood CE. A program to increase the number of family physicians in rural and underserved areas: impact after 22 years. JAMA. 1999;281(3):255-60.

23. Laurence CO, Williamson V, Summer KE, Fleming J. "Latte rural": the tangible and intangible factors important in the choice of a rural practice by recent GP graduates. Rural Remote Health. 2010;10(2):1316.

24. Shanley BC, Schulte KM, Chant D, Jasper A, Wellard R. Factors influencing career development of Australian general practitioners. Aust Fam Physician. 2002;31(1):4954.

25. Anderson J. Tendiendo puentes: calidad de atención desde la perspectiva de las mujeres rurales y de los proveedores de los servicios de salud. Lima: Movimiento Manuela Ramos; 2001.

26. Reyes E. En nombre del Estado. Servidores públicos de una microrred de salud en la costa rural del Perú. Lima: UPCH/IEP; 2007

27. Vanasse A, Scott S, Courteau J, Orzanco MG. Canadian family physicians' intentions to migrate: associate factors. Can Fam Physician. 2009;55(4):396-97.

28. Pardo K, Andia M, Rodríguez A, Pérez W, Moscoso B. Remuneraciones, beneficios e incentivos laborales percibidos por trabajadores del sector salud en el Perú: análisis comparativo entre el Misniterio de Salud y la Seguridad Social, 2009. Rev Peru Med Exp Salud Publica. 2011;28(2):342-51.

29. Willis-Shattuck M, Bidwell $P$, Thomas $S$, Wyness $L$, Blaauw D, Ditlopo P. Motivation and retention of health workers in developing countries: a systematic review. BMC Health Serv Res. 2008;8:247.

30. Romero-Escalante L, Cueva-Chávez L, Linares-Reyes E, Blossiers-Mazzini C. Experiencias de trabajo en actividades en atención primaria de salud realizadas por estudiantes de medicina de Perú. CIMEL. 2010;15(1):14-8.

31. Rivas-Nieto AC, Curioso WH, Guillén C. Participación estudiantil en proyectos de intervención rural en salud: La experiencia IRIS-X en Perú. Rev Peru Med Exp Salud Publica. 2009;26(3):387-94.

Correspondencia: Dr. Percy Mayta-Tristán

Dirección: Av. Brasil 2169, Dpto 802, Lima 11, Perú.

Teléfono: (511) 987-532133

Correo electrónico: percy.mayta@upc.edu.pe,

p.mayta@gmail.com 